\title{
Descriptive statistics (Part 2): Interpreting study results
}

\author{
A Cook and A Sheikh
}

\section{Adrian Cook}

Statistician

Aziz Sheikh

Clinical Research Fellow

Department of Primary Health Care and General Practice, Imperial College School of Medicine, Norfolk Place, London W2 1PG, UK

Correspondence to: Mr A Cook

a.d.cook@ic.ac.uk

Date received: 26/11/99 Date accepted: 01/02/00

Asthma Gen Pract 2000;8(1):16-17
$\mathrm{T}$ he previous paper in this series looked at descriptive statistics, showing how to use and interpret fundamental measures such as the mean and standard deviation. Here we continue with descriptive statistics, looking at measures more specific to medical research. We start by defining risk and odds, the two basic measures of disease probability. Then we show how the effect of a disease risk factor, or a treatment, can be measured using the relative risk or the odds ratio. Finally we discuss the 'number needed to treat', a measure derived from the relative risk, which has gained popularity because of its clinical usefulness. Examples from the literature are used to illustrate important concepts.

\section{RISK AND ODDS}

The probability of an individual becoming diseased is the risk. An example comes from a survey of four factories that used acid anhydrides, ${ }^{1}$ workers were asked about respiratory problems beginning after the start of employment, results shown in Table 1. Respiratory symptoms were reported by 34 of the 401 subjects, the risk therefore being $34 / 401=0.085$, or $8.5 \%$. In other words, among 100 factory workers exposed to acid anhydrides, eight or nine would be expected to develop respiratory symptoms.

Table 1: Number of acid anhydride workers developing respiratory symptoms

Work-related respiratory symptoms

\begin{tabular}{llll}
\hline & Yes (case) & No (non-case) & Total \\
\hline $\mathrm{N}$ & 34 & 367 & 401
\end{tabular}

The concept of odds is familiar to gamblers as the ratio between amounts at stake in a bet, odds of $4: 1$ mean that if one party stakes $£ 4$ and the other stakes $£ 1$, then the winner takes the whole $£ 5$. Odds of disease is the ratio between the probability of disease and the probability of no disease. From surveys, it is estimated by the number of cases divided by the number of noncases. The odds of a factory worker exposed to acid anhydrides developing respiratory symptoms is $34 / 367=0.092$, slightly higher than the risk.

Rare diseases yield similar risk and odds since the number of non-cases is close to the number of subjects. For common diseases, risk and odds can differ greatly and it is thus important to differentiate between the two.

\section{RELATIVE RISK AND ODDS RATIO}

The workers represented in Table 1 were employed at four different factories, with factory two known to use large amounts of trimellitic anhydride (TMA). To investigate the relative danger of this particular chemical the risk or odds of workers at factory two can be compared with workers at the other factories. Workers at the other factories are referred to as 'unexposed', and their risk or odds is referred to as 'baseline'. Investigations of treatment effects can be made in similar fashion by comparisons of disease probability in treated and untreated patients.

The relative risk (RR), also sometimes known as the risk ratio, compares the risk of exposed and unexposed subjects, while the odds ratio (OR) compares odds. A relative risk or odds ratio greater than one indicates an exposure to be harmful, while a value less than one indicates a protective effect. $\mathrm{RR}=1.2$ means exposed people are $20 \%$ more likely to be diseased, $\mathrm{RR}=1.4$ means $40 \%$ more likely. $\mathrm{OR}=1.2$ means that the odds of disease is $20 \%$ higher in exposed people.

Among workers at factory two ('exposed' workers) the risk is $13 / 116=0.11$, compared to an 'unexposed' risk of $21 / 285=0.07$ (Table 2 ). The relative risk is therefore $0.11 / 0.07=1.52$, indicating that workers exposed to TMA are about $50 \%$ more likely to develop respiratory symptoms than workers exposed to other anhydrides. A similar calculation with odds gives an odds ratio of 1.59 , slightly higher than the RR.

Table 2: Work-related respiratory symptoms, by TMA exposure

Work-related respiratory symptoms

\begin{tabular}{llll}
\hline & Yes & No & \\
\hline Exposed & 13 & 103 & 116 \\
Not exposed & 21 & 264 & 285 \\
\hline & 34 & 367 & 401
\end{tabular}

\section{Relative risk vs odds ratio}

Relative risk is more easily understood than the odds ratio, for this reason it can be regarded as the better of the two measures from a practitioner's viewpoint. Odds ratios are, however, widely used because of situations in which they have mathematical advantages (see below). The odds ratio can be regarded as an estimate of the relative risk when disease risk is low in both groups, say $20 \%$ or less. The approximation becomes less valid as baseline risk or effect size increases. ${ }^{2}$

Odds ratios are commonly used in multivariate analyses, when effect estimates need to be adjusted for factors such as age which may differ between the two groups. They are also used to analyse casecontrol studies, an epidemiological study design, which retrospectively compares 'cases' of disease with healthy 'controls'.

The relative risk and odds ratio are both relative measures of effect, as such they are unaffected by changes in baseline risk. In other words, studies carried out in different regions with different disease levels should give the same result, the strength of relative measures lies in this 'portability'. 
Unfortunately they give no indication of just how many people are affected. For a given relative risk, more cases will occur if the associated disease is common than if it is rare. Therefore, understanding the implications of RRs and ORs in public health terms requires baseline risk to be considered.

\section{NUMBER NEEDED TO TREAT}

The number needed to treat (NNT) combines the relative risk and baseline risk in a single clinically meaningful statistic. NNT is the number of patients requiring treatment for one extra successful outcome. As an example, if $30 \%$ of patients in the placebo group of a randomised trial recover and $40 \%$ recover in the treated group, by treating ten patients four will recover instead of three, so one extra successful outcome occurs and NNT $=10$. The NNT can be calculated as the inverse of the risk difference, in this case 1/(0.4-0.3), however the formula given in Table 4 is generally easier to apply. In terms of risk factors, NNT represents the number of people who must be removed from exposure to prevent one case of disease. Problems can occur when calculating confidence intervals for the NNT, ${ }^{3}$ to overcome this the number needed to benefit (NNTB) and the number needed to harm (NNTH) have been developed. The NNTB and NNTH correspond to relative risks greater and less than one respectively.

Data from a clinical trial of smoking cessation are given in Table $3 .^{4}$ Participants received nicotine patches and either a nicotine nasal spray or placebo spray for one year. Six years later, $16.1 \%$ of those given active sprays were abstinent from smoking, compared with $8.4 \%$ of the placebo group. From the placebo group, the baseline 'risk' of stopping is 0.084 . For those receiving nicotine spray, the relative risk is $0.161 / 0.084=1.92$, therefore they are almost twice as likely to succeed. The number needed to treat is 13, calculated from the formula given in Table 4; of 13 patients receiving nicotine nasal spray in addition to a patch, one is expected to give up smoking who would not otherwise have done so.

Table 3: Percentages smoking at six year follow up, by treatment group

Smoking status $(n=237)$

\begin{tabular}{lcll}
\hline & No \% $(n)$ & \multicolumn{2}{l}{ Yes \% (n) } \\
\hline Patch and nicotine spray & $16.1(19)$ & 83.9 & $(99)$ \\
Patch and placebo spray & $8.4(10)$ & $91.6 \quad(109)$
\end{tabular}

Table 4: Important definitions

\begin{tabular}{ll} 
Measure & Definition \\
\hline Risk & $\begin{array}{l}\text { Number of cases/Number } \\
\text { of subjects }\end{array}$ \\
\hline Odds & $\begin{array}{l}\text { Number of cases/Number of } \\
\text { non-cases }\end{array}$ \\
\hline Relative risk (RR) & Risk in exposed/Risk in unexposed \\
\hline Odds ratio (OR) & $\begin{array}{l}\text { Odds in exposed/Odds in } \\
\text { unexposed }\end{array}$ \\
\hline $\begin{array}{l}\text { Number needed } \\
\text { to treat }\end{array}$ & \begin{tabular}{l} 
1/ (Risk in unexposed*(RR-1)) \\
\hline
\end{tabular}
\end{tabular}

The number needed to treat is now frequently reported in trial results. When not given it can be calculated from the RR and baseline risk. The odds ratio may be used in place of the relative risk when the risk in both groups is low.

\section{CONCLUSION}

Relative risks and odds ratios are used in epidemiology to describe the effect of risk factors, and in trials to describe the effect of treatment. The strength of both measures is that they are unaffected by changes in baseline prevalence and hence 'portable'. Their major drawback lies in the difficulty of interpretation in a clinical context. The number needed to treat overcomes this weakness and has been an important step in bridging the gap between research findings and understanding their clinical significance.

\section{Acknowledgements}

We thank Bernadette Alves, Pauline Bryant, Sangeeta Dhami and John Salinsky for their constructive comments on earlier drafts of this manuscript.

\section{References}

1. Barker RD, van Tongeren MJA, Harris JM, et al. Risk factors for sensitisation and respiratory symptoms among workers exposed to acid anhydrides: A cohort study. Occup Environ Med 1998;55:684-91

2. Davies HTO, Crombie IK, Tavakoli M. When can odds ratios mislead? BMJ 1998;316:989-91

3. Altman DG. Confidence intervals for the number needed to treat. BMJ 1998; 317:1309-12

4. Blondal T, Jon Gudmundsson J, Olafsdottir I, et al. Nicotine nasal spray with nicotine patch for smoking cessation: randomised trial with six year follow up. BMJ 1999;318:285-8 